\title{
An Initial-Boundary Value Problem for the Viscous Compressible Flow
}

\author{
Friedrich-Karl Hebeker* George C. Hsiao ${ }^{\dagger}$ \\ In Memory of Gaetano Fichera
}

\begin{abstract}
A constructive approach is presented to treat an initial boundary value problem for isothermal Navier Stokes equations. It is based on a characteristics (Lagrangean) approximation locally in time and a boundary integral equation method via nonstationary potentials. As a basic problem, the later leads to a Volterra integral equation of the first kind which is proved to be uniquely solvable and even coercive in some anisotropic Sobolev spaces. The solution depends continuously upon the data and may be constructed by a quasioptimal Galerkin procedure.
\end{abstract}

AMS: 76M15, 76M30, 76N10, 45D05, 35K05

KEYWORDS: boundary integral equations, fundamental solution, coercivity, Korn's inequality, anisotropic Sobolev spaces, variational formulation, weak solution.

\section{Introduction}

In this paper, we are concerned with a boundary integral equation approach to treat problems of non-stationary isothermal viscous compressible flow.

*Fachbereich Mathematik, Universität Duisburg-Essen, 45117 Essen, Germany (Email: fk.hebeker@tiscali.de).

${ }^{\dagger}$ Department of Mathematical Sciences, University of Delaware, Newark, Delaware 19716, USA (Email: hsiao@math.udel.edu). 
For definiteness, let us begin with the initial boundary value problem of the isothermal Navier-Stokes equations governing the flow in a bounded cavity $\Omega \subset \mathbb{R}^{n}(n=2,3)$ with smooth boundary $\partial \Omega$ :

$$
\begin{gathered}
\rho\left(v_{t}+v \cdot \nabla v\right)-\frac{1}{R e}\left(\Delta v+\frac{1}{3} \nabla \operatorname{div} v\right)+\nabla p(\rho)=0 \\
\rho_{t}+v \cdot \nabla \rho+\rho \operatorname{div} v=0
\end{gathered}
$$

together with boundary and initial conditions:

$$
\left.v\right|_{\partial \Omega}=g,\left.\quad \rho\right|_{t=0}=\rho_{0},\left.\quad v\right|_{t=0}=v_{0} .
$$

Here $v(x, t)$ denotes the velocity field, $p(x, t)$ the pressure field, and $\rho(x, t)$ the density function. The flow is assumed to be isentropic, that is, the pressure field $p(x, t)$ is a function of $\rho$ according to the relation

$$
p(\rho)=\frac{c_{0}}{\kappa M^{2}} \rho^{\kappa},
$$

where $c_{0}$ is a constant, $\kappa(\geq 1)$ denotes the adiabatic exponent (e.g., $\kappa=1.4$ for air at standard sea-level), and $M$ is the Mach number, while Re denotes the Reynolds number of the flow.

Problem (1.1)-(1.2) may be simplified by means of a semi-implicit method of time discretization on characteristic (Lagrangean) coordinates (see, e.g., [5]). Assuming that the density does not vary too much, we may then determine the approximate velocity field (on small time intervals) from the linearized parabolic differential system:

$$
\begin{gathered}
L v:=v_{t}-\mu \Delta v-\nu \nabla \operatorname{div} v=f \\
\left.v\right|_{\partial \Omega}=g,\left.\quad v\right|_{t=0}=v_{0} .
\end{gathered}
$$

Alternatively, (1.4) can be obtained from (1.1), by assuming (relatively) small velocities of the flow with $\mu=1 / \bar{\rho} R e$, and $\nu=1 / 3 \mu$ where

$$
\bar{\rho}=\frac{1}{|\Omega|} \int_{\Omega} \rho_{0} d x
$$

denotes the mean density of the flow, while the nonhomogeneous term $f$ contains further information about the density (assumed as - approximately 
- known). Then the approximate density in turn is obtained in term of Lagrangean coordinates from the linearized equation of continuity

$$
\begin{gathered}
\rho_{t}+\rho \operatorname{div} v=0 \\
\left.\rho\right|_{t=0}=\rho_{0}
\end{gathered}
$$

(on small time steps), which is easily solvable for any given $v$. This reduction by approximation on short time interval is justified by employing the convergence analysis of [5]: the procedure is first order consistent, and is convergent as long as the computations remain stable (in a rather strong sense).

Through out of the paper, we shall refer to the linearized parabolic differential system (1.4)-(1.5) as the basic model problem for the viscous compressible flow. Our aim is to provide for a constructive approach to solve the basic model problem (1.4)-(1.5) via the boundary integral equation method. The paper is organized as follows: In Section 2, we construct the fundamental solution of (1.4) in term which we derive the corresponding Green formula for (1.4). Section 3 contains the representation of the solution for the basic problem defined by (1.4) and (1.5) as a sum of a volume, PoissonWeierstrass, simple- and double-layer potentials. This reduces the problem to an equivalent first kind boundary integral equation of the Volterra type. The main result of the present paper is stated in Theorem 1 concerning the unique solvability of this integral equation. In fact, it is shown that the corresponding integral operator is even elliptic in some anisotropic Sobolev spaces. The later implies the quasioptimality of the appropriate Galerkin approximations. These results extend now the standard variational techniques for steady-state strongly elliptic boundary integral equations as in [3] and [8] to the unsteady viscous compressible flow case, by employing the tools due as in [1], [2] and [7] for the heat equations. The proofs are given in Section $4-6$.

\section{Green's Representation Formula}

System (1.4) forms a (strong coupled) parabolic system in the sense of Petrovskij. It does not appear very often in the well known classical mathematical physics, but it does play an important role for the method of artificial compressibility in computational incompressible flows (see, e.g., [4]). Hence a potential theory approach to (1.4) would be valuable. 
Let $\Gamma_{\mu}$ denote the fundamental solution for the heat operator $\left(\partial_{t}-\mu \Delta\right)$ :

$$
\Gamma_{\mu}= \begin{cases}(4 \pi \mu t)^{-n / 2} \exp \left(-|x|^{2} /(4 \mu t)\right) & : t>0 \\ 0 & : t \leq 0\end{cases}
$$

In terms of $\Gamma_{\mu}$, the fundamental solution of the system (1.4) is a matrix function $\Gamma_{i j}$ of the form:

$$
\Gamma_{i j}(x, t)=\delta_{i j} \Gamma_{\mu}(x, t)+\frac{\partial^{2}}{\partial x_{i} \partial x_{j}} \gamma(x, t) \quad(t>0, x \neq 0),
$$

where $\gamma$ is a scalar function satisfying the inhomogeneous heat equation up to $t=0$ (for any $x \in \mathbb{R}^{n}$ ):

$$
\gamma_{t}-(\mu+\nu) \Delta \gamma=\nu \Gamma_{\mu}
$$

In fact, we may assume that $\gamma$ is radially symmetric, and based on the argument of homogeneity it suggests that $\gamma$ may be of the from $\gamma(x, t)=$ $t^{1-n / 2} w\left(r^{2} / t\right)$, where $w(z)$ with $z=r^{2} / t$ satisfies the ordinary differential equation

$$
4(\mu+\nu) z w^{\prime \prime}+(z+2(\mu+\nu) n) w^{\prime}+\left(\frac{n}{2}-1\right) w=\nu(4 \pi \mu)^{-n / 2} e^{-z /(4 \mu)} .
$$

In case $n=2$, we have

$$
\gamma(r, t)=\frac{1}{4 \pi} \int_{r^{2} / t}^{\infty} \frac{1}{z} e^{-z /(4 \mu)} d z
$$

Multiplying (1.4) by $v$ and integrating by parts, we then obtain the second Green identity (for sufficiently smooth $u, v$ )

$$
\begin{gathered}
\int_{0}^{t} \int_{\Omega}\left\{\left(u_{s}-\mu \Delta u-\nu \nabla \operatorname{div} u\right) \cdot v-u \cdot\left(-v_{s}-\mu \Delta v-\nu \nabla \operatorname{div} v\right)\right\} d y d s \\
=\int_{0}^{t} \int_{\Omega}(u \cdot v)_{s} d y d s-\int_{0}^{t} \int_{\partial \Omega} \sigma(u) \cdot v-u \cdot \sigma(v) d o_{y} d s
\end{gathered}
$$

where $\hat{n}$ denotes the outward unit normal vector, and

$$
\sigma(u):=\nu(\operatorname{div} u) \hat{n}+\mu\left(\nabla u+\nabla u^{\top}\right) \hat{n}
$$


(the superscript ${ }^{\top}$ denotes the transpose). Substituting now $v$ by the column vector $\Gamma_{j}$ of the symmetric fundamental matrix $\Gamma_{i j}$,

$$
v(y, s)=\Gamma_{j}(x-y, t-s) \quad(j=1,2,3)
$$

and using the standard argument in potential theory, we obtain the Green representation formula

$$
\begin{aligned}
u_{j}(x, t) & =\int_{0}^{t} \int_{\Omega} \Gamma_{j}(x-y, t-s) \cdot\left(u_{s}-\mu \Delta u-\nu \nabla d i v u\right)(y, s) d y d s \\
& +\int_{\Omega} \Gamma_{j}(x-y, t) u(y, 0) d y+\int_{0}^{t} \int_{\partial \Omega} \Gamma_{j}(x-y, t-s) \sigma(u)(y, s) d o_{y} d s \\
& -\int_{0}^{t} \int_{\partial \Omega} \sigma_{y}\left(\Gamma_{j}(x-y, t-s)\right) \cdot u(y, s) d o_{y} d s \quad(j=1,2,3)
\end{aligned}
$$

In an analog to the potential theory for the elliptic equations, we now represent any smooth solution of the basic model problem (1.4)-(1.5) in the form:

$$
v=N f+P v_{0}+V\left(\left.\sigma(v)\right|_{\partial \Omega}\right)-W g,
$$

where $N, P, V, W$ denote the volume, Poisson-Weierstrass, simple - and doublelayer potentials, respectively. Here the operators are defined in a consecutive order according to those in (2.7).

We note that although the formula (2.8) here is derived for the bounded domain $\Omega$, it may be easily extended to unbounded domains with slight modification.

\section{Boundary Integral Equation of the First Kind}

We note that the only unknown appearing on the right hand side of $(2.8)$ is the vector field $\left.\sigma(v)\right|_{\partial \Omega}$. For continuous vector fields $\phi$ on the boundary one can show by classical means (see, e.g., [9]) that the simple-layer potential $u=V \phi$ is continuous over the whole space for $t>0$. Consequently, from the representation formula (2.8), if we denote the unknown field $\sigma(v)$ by $\psi$, then we obtain the boundary integral equation of the first kind for $\psi$ :

$$
A \psi=b \quad \text { on } \quad \partial \Omega_{T}=(0, T) \times \partial \Omega,
$$


as $x \in \Omega$ approaches to the boundary $\partial \Omega$, where $A=\gamma V$ ( $\gamma$ the trace on $\partial \Omega$ ), and $b$ is the collection of all the known quantities in (2.8). Hence our aim here is to investigate the solvability of the equation (3.1). We now extend to the equation (3.1) those approaches in [1], [2] and [7] for treating heat equation.

To this end, let us introduce the anisotropic Sobolev spaces ([11], Vol 2)

$$
H^{r, s}\left(\partial \Omega_{T}\right)=L^{2}\left(0, T, H^{r}(\partial \Omega)\right) \cap H^{s}\left(0, T, L^{2}(\partial \Omega)\right) \quad(r, s \geq 0),
$$

where $(0, T)$ denotes a finite time interval and $H^{r}$ the usual Sobolev-Slobodetski spaces obtained by interpolation between $H^{m}$ and $L^{2}$ (m, integer). We also need

$$
H_{, 0}^{r, s}\left(\partial \Omega_{T}\right)=L^{2}\left(0, T, H^{r}(\partial \Omega)\right) \cap H_{0}^{s}\left(0, T, L^{2}(\partial \Omega)\right) \quad(r, s \geq 0) .
$$

Here $H^{r, s}$ and $H_{, 0}^{r, s}$ are Hilbert spaces equipped with the natural norm:

$$
\|\phi\|_{r, s}=\left(\|\phi\|_{L^{2}\left(0, T, H^{r}(\partial \Omega)\right)}^{2}+\|\phi\|_{H^{s}\left(0, T, L^{2}(\partial \Omega)\right)}^{2}\right)^{1 / 2},
$$

or alternatively written as

$$
\|\phi\|_{r, s}=\left(\|\phi\|_{r, 0}^{2}+\|\phi\|_{0, s}^{2}\right)^{1 / 2} .
$$

For negative exponents we denote by $H^{-r,-s}\left(\partial \Omega_{T}\right)=\left(H_{, 0}^{r, s}\left(\partial \Omega_{T}\right)\right)^{*}$ the dual spaces of $H_{, 0}^{r, s}\left(\partial \Omega_{T}\right)$, equipped with the norm

$$
\|\phi\|_{-r,-s}=\sup _{\psi \in H_{, 0}^{r, s}} \frac{<\phi, \psi>}{\|\psi\|_{r, s}} \quad(r, s \geq 0, r \text { or } s \text { positive })
$$

where $\langle\cdot, \cdot\rangle$ is the duality pairing between $H^{-r,-s}$ and $H_{, 0}^{r, s}$. We adopt the notations here for the scalar as well as for the vector fields, and we employ the similar notations for functions on $\Omega_{T}$ with $\partial \Omega$ replaced by $\Omega_{T}$ in the above definitions.

We are now in a position to state a crucial result of the paper.

Proposition 1 For any $\lambda>0$, the operator $e^{-\lambda t} A$ defines a mapping

$$
e^{-\lambda t} A: H^{-1 / 2,-1 / 4}\left(\partial \Omega_{T}\right) \mapsto H^{1 / 2,1 / 4}\left(\partial \Omega_{T}\right)
$$

which is continuous and coercive. Here the energy space $H^{-1 / 2,-1 / 4}\left(\partial \Omega_{T}\right)$ is just the dual of $H^{1 / 2,1 / 4}\left(\partial \Omega_{T}\right)$. 
This result will be proved in Section 4-5. It plays a fundamental role for the subsequent main theorem, since it implies immediately that the integral operator $A$ in (3.1) is also an isomorphism. From this, in turn, we obtain by use of the standard variational arguments (as in [8]) to establish the coercivity of A. We will carry out the detailed analysis in Section 6 .

Theorem 1 The operator

$$
A: H^{-1 / 2,-1 / 4}\left(\partial \Omega_{T}\right) \mapsto H^{1 / 2,1 / 4}\left(\partial \Omega_{T}\right)
$$

is continuous and coercive. Consequently, the Volterra integral equation (3.1) in weak form

$$
<\phi, A \psi>=<\phi, b>\quad \text { for all } \quad \phi \in H^{-1 / 2,-1 / 4}\left(\partial \Omega_{T}\right)
$$

has a unique solution $\psi \in H^{-1 / 2,-1 / 4}\left(\partial \Omega_{T}\right)$, depending continuously on the data $b \in H^{1 / 2,1 / 4}\left(\partial \Omega_{T}\right)$.

This theorem implies via Céa's lemma the quasi-optimality of any Galerkin scheme to solve the basic model problem defined by (1.4)-(1.5) approximately on small time intervals $[0, \tau]$. More precisely, let

$$
H_{h} \subset H^{-1 / 2,-1 / 4}\left(\partial \Omega_{T}\right), \quad h \in\left(0, h_{0}\right]
$$

be a family of finite-dimensional subspaces. Then the Galerkin approximate $\psi_{h} \in H_{h}$ is a solution of the Galerkin equations

$$
<\chi, A \psi_{h}>=<\chi, b>\text { for all } \quad \chi \in H_{h} .
$$

The quasioptimality follows immediately from the coercivity of $A$ from Theorem 1:

$$
\left\|\psi_{h}-\psi\right\|_{-1 / 2,-1 / 4} \leq \text { const. } \inf _{\chi \in H_{h}}\|\chi-\psi\|_{-1 / 2,-1 / 4} .
$$

In practice, to implement (3.10), one usually uses tensor products

$$
\chi_{i}(x, t)=p_{i}(t) q_{i}(x), \quad \text { where } \quad t \in(0, \tau), x \in \partial \Omega,
$$

of low order polynomials $p_{i}$ and piecewise polynomials $q_{i}$ lifted to the parameter space of the boundary, subordinated to a given decomposition of $\partial \Omega$ into boundary elements. 


\section{Continuity of $e^{-\lambda t} A$}

In the next two sections we shall prove Proposition 1. We begin with the continuity. Since we are concerned with the finite time intervals, it is more convenient to reformulate the problem (3.1) in the form

$$
A^{\prime} \psi^{\prime}=b^{\prime} \quad \text { on } \quad \partial \Omega \times \mathbb{R},
$$

where $b^{\prime}=e^{-\lambda t} b$ on $(0, T)$ and $b^{\prime}=0$ elsewhere. Here $A^{\prime}=\gamma V^{\prime}$ and the simple-layer potential $V^{\prime}$ corresponds to the differential operator

$$
L^{\prime} u:=u_{t}-\mu \Delta u-\nu \nabla \operatorname{div} u+\lambda u \quad \text { on } \quad \mathbb{R}_{\infty}^{n}=\mathbb{R}^{n} \times \mathbb{R} .
$$

The formula

$$
L e^{\lambda t}=e^{\lambda t} L^{\prime}
$$

follows by definition (see (1.4)). In what follows, it is understood that the constant $\lambda$ is fixed and positive.

The trace operator $\gamma$ is a continuous map

$$
\gamma: H^{1,1 / 2}\left(\mathbb{R}_{\infty}^{n}\right) \rightarrow H^{1 / 2,1 / 4}\left(\partial \Omega_{\infty}\right)
$$

(see [11],Vol.2, 9f). Hence the adjoint operator is a continuous map

$$
\gamma^{*}: H^{-1 / 2,-1 / 4}\left(\partial \Omega_{\infty}\right) \rightarrow H^{-1,-1 / 2}\left(\mathbb{R}_{\infty}^{n}\right),
$$

which extends the inner product of $L^{2}\left(\partial \Omega_{\infty}\right)$ to elements $\phi \in H^{-1 / 2,-1 / 4}\left(\partial \Omega_{\infty}\right)$ such that

$$
<\gamma^{*} \phi, w>=<\phi, \gamma w>=\int_{\mathbb{R}} \int_{\partial \Omega} \phi \cdot w d o d t \text { for all } w \in H^{1,1 / 2}\left(\mathbb{R}_{\infty}^{n}\right) .
$$

This suggests to identify the simple-layer potential of density $\phi$ with the volume potential of density $\gamma^{*} \phi$ :

$$
V^{\prime} \phi=N^{\prime} \gamma^{*} \phi
$$

By interpreting the volume potential as inverse of the operator $L^{\prime}$ in (4.2) on $\mathbb{R}_{\infty}^{n}$, the boundary integral operator $A^{\prime}$ may be factorized as

$$
A^{\prime}=\gamma L^{\prime-1} \gamma^{*} \text {. }
$$

Hence the continuity is a consequence of the following lemma. 
Lemma 1 The mapping defined by the operator $L^{\prime}$ :

$$
L^{\prime}: H^{1,1 / 2}\left(\mathbb{R}_{\infty}^{n}\right) \rightarrow H^{-1,-1 / 2}\left(\mathbb{R}_{\infty}^{n}\right)
$$

is an isomorphism.

Proof. Let $W$ denote the space

$$
W:=H^{1,0}\left(\mathbb{R}_{\infty}^{n}\right) \cap H^{1}\left(\mathbb{R}, H^{-1}\left(\mathbb{R}^{n}\right)\right) .
$$

We divide the proof into following three steps:

First, we show that

$$
L^{\prime}: W \rightarrow H^{-1,0}\left(\mathbb{R}_{\infty}^{n}\right)
$$

is an isomorphism. We proceed as follows. Consider the formal adjoint of $L^{\prime}$ :

$$
L^{*} v=-v_{t}-\mu \Delta v-\nu \nabla \operatorname{div} v+\lambda v
$$

and evidently the mapping $L^{*}: W \rightarrow H^{-1,0}$ is continuous. With $\beta=$ $\min \{\mu, \lambda\}>0$, the energy estimate

$$
<L^{*} v, v>\geq \beta\|v\|_{1,0}^{2} \text { for all } \quad v \in W .
$$

is easily shown. Hence from a variant of the Lax-Milgram projection lemma [10], it follows that for any $f \in H^{-1,0}$, there is a unique solution $u \in H^{1,0}$ of the equation

$$
<L^{*} v, u>=<f, v>\text { for all } v \in W .
$$

Hence $u_{t}$ exists in the sense of distributions and, since $\bar{W}=H^{1,0}$, we see that

$$
u_{t}=f+\mu \Delta u+\nu \nabla \operatorname{div} u-\lambda u \in H^{-1,0} .
$$

Consequently, $u \in W$ and we obtain the estimate

$$
\|u\|_{W} \leq \text { const. }\|f\|_{-1,0}
$$

from Lions' projection lemma. This completes the proof of (4.9).

Next, we extend $L^{\prime}$ to $H^{1,0}$ by transposition:

$$
L^{\prime} u=f \quad \text { in } \quad W^{*} \Leftrightarrow<L^{*} v, u>=<f, v>\quad \text { for all } \quad v \in W .
$$

Here we will show that the mapping

$$
L^{\prime}: H^{1,0}\left(\mathbb{R}_{\infty}^{n}\right) \rightarrow W^{*}
$$


is an isomorphism. Clearly the mapping $L^{\prime}: H^{1,0} \rightarrow W^{*}$ is continuous. Moreover, we already know that

$$
T: W \rightarrow H^{-1,0} \quad\left(\text { with } \quad v \mapsto<L^{*} v, \cdot>\right)
$$

is an isomorphism and hence the adjoint map

$$
T^{*}: H^{1,0} \rightarrow W^{*} \quad\left(\text { with } \quad u \mapsto<L^{*} \cdot, u>\right)
$$

is also an isomorphism. Then for any given $f \in W^{*}$, there is a unique solution $u \in H^{1,0}$ of the equation

$$
<L^{*} v, u>=<f, v>\text { for all } \quad v \in W .
$$

Moreover, $\|u\|_{1,0} \leq$ const. $\|f\|_{W^{*}}$ holds. That is, (4.15) is verified.

Finally, we see that (4.7) now follows by interpolation between (4.9) and (4.15). We have

$$
\begin{aligned}
{\left[W, H^{1,0}\right]_{1 / 2} } & =\left[H^{1,0} \cap H^{1}\left(\mathbb{R}, H^{-1}\left(\mathbb{R}^{n}\right)\right), H^{1,0}\right]_{1 / 2} \\
& =H^{1,0} \cap\left[H^{1}\left(\mathbb{R}, H^{-1}\left(\mathbb{R}^{n}\right)\right), H^{1,0}\right]_{1 / 2} \\
& =H^{1,0} \cap H^{0,1 / 2}=H^{1,1 / 2}\left(\mathbb{R}_{\infty}^{n}\right)
\end{aligned}
$$

([11], Vol.1, p.233 and p.255). On the other hand, from ([11], Vol.1, p.29), we see that

$$
\left[H^{-1,0}, W^{*}\right]_{1 / 2}=\left(\left[W, H^{1,0}\right]_{1 / 2}\right)^{*}=H^{-1,-1 / 2}\left(\mathbb{R}_{\infty}^{n}\right) .
$$

The Interpolation Theorem ([11], Vol.1, p.27) implies (4.7), and this completes the proof of Lemma 1.

A collection of (4.6), (4.5), (4.7) and (4.4) yields

$$
A^{\prime}: H^{-1 / 2,-1 / 4}\left(\partial \Omega_{\infty}\right) \rightarrow H^{1 / 2,1 / 4}\left(\partial \Omega_{\infty}\right) \quad \text { continuously. }
$$

As can be seen, this implies the continuity of $A$ and hence of $e^{-\lambda t} A$. The later can be seen as follows. Let $E$ denotes the extension by zero in time, and $R$ be the restriction to the interval $(0, T)$. Clearly $R=E^{*}$ is valid, and from (4.3), we may write

$$
L^{-1} e^{\lambda t}=e^{\lambda t} L^{\prime-1} .
$$

The continuity of $A$ then follows from the composition

$$
A=R \gamma L^{-1} \gamma^{*} E=e^{\lambda t} R A^{\prime} E e^{-\lambda t}
$$

(see (4.6)). Hence we have shown that

$$
A: H^{-1 / 2,-1 / 4}\left(\partial \Omega_{T}\right) \rightarrow H^{1 / 2,1 / 4}\left(\partial \Omega_{T}\right) \text { continuously, }
$$

and the same holds for $e^{\lambda t} A$. 


\section{Coercivity of $e^{-\lambda t} A$}

We begin with the operator $A^{\prime}$ in (4.6) and we need first the lemma.

Lemma 2 Let $D \subset \mathbb{R}^{n}$ be a bounded domain with smooth boundary $\partial D$. Then to any $f \in H^{-1,0}\left(D_{\infty}\right) \cap\left(H^{1,1 / 2}\left(D_{\infty}\right)\right)^{*}$ and $g \in H^{1 / 2,1 / 4}\left(\partial D_{\infty}\right)$, the problem

$$
u_{t}-\Delta u=f \quad \text { in } \quad D_{\infty}, \quad \gamma u=g \quad \text { on } \quad \partial D_{\infty}
$$

has a unique solution

$$
u \in W\left(D_{\infty}\right)=H^{1,0}\left(D_{\infty}\right) \cap H^{1}\left(\mathbb{R}, H^{-1}(D)\right)
$$

and the solution depends continuously upon the (vector) data.

This is a variant of assertion (15.38) stated in [11](Vol.2, p.84) without a complete proof. We will include the proof below in four steps:

(1) We will show that for any $f \in L^{2}\left(D_{\infty}\right)$ and $g \in H^{3 / 2,3 / 4}\left(\partial D_{\infty}\right)$, there is a unique solution $u \in H^{2,1}\left(D_{\infty}\right)$ of (5.1), depending continuously on the data. For this, the Trace Theorem ([11], Vol.2, p.9) guarantees that there exists a function $v \in H^{2,1}\left(D_{\infty}\right)$ with $\gamma v=g$ such that the difference $w=v-u$ satisfies

$$
w_{t}-\Delta w=\phi:=v_{t}-\Delta v-f \in H^{0,0}\left(D_{\infty}\right) ; \quad \gamma w=0
$$

in the infinite cylinder $D_{\infty}$. By applying the Fourier transform of (5.3) with respect to $t$ and making use of the Agmon-Nirenberg estimate of the transformed function $\hat{w}$, we obtain

$$
\begin{aligned}
\|w\|_{2,0}^{2} & =\|\hat{w}\|_{2,0}^{2}=\int_{\mathbb{R}}\left\|(i \tau-\Delta)^{-1} \hat{\phi}(\cdot, \tau)\right\|_{H^{2}(D)}^{2} d \tau \\
& \leq \text { const. } \int_{\mathbb{R}}\|\hat{\phi}(\cdot, \tau)\|_{L^{2}(D)}^{2} d \tau=\text { const. }\|\phi\|_{0,0}^{2} .
\end{aligned}
$$

Hence from the differential equation (5.3)

$$
\|w\|_{2,1} \leq \text { const. }\|\phi\|_{0,0}
$$

follows and this implies the desired result

$$
\|u\|_{2,1} \leq\|v\|_{2,1}+\|w\|_{2,1} \leq \text { const. }\left(\|f\|_{0,0}+\|g\|_{3 / 2,3 / 4}\right) .
$$


(2) By transposition of the problem adjoint to that treated in step (1), we get a unique weak solution $u \in L^{2}\left(D_{\infty}\right)$ of (5.1) for any $f \in\left(H^{2,1}\left(D_{\infty}\right)\right)^{*}$ and $g \in\left(H^{1 / 2,1 / 4}\left(\partial D_{\infty}\right)\right)^{*}$. Again, $u$ depends continuously upon the data.

(3) By interpolation between the isomorphisms in steps (1) and (2), we see that for any

$$
\begin{aligned}
f \in\left(H^{1,1 / 2}\left(D_{\infty}\right)\right)^{*} & =\left(\left[H^{2,1}\left(D_{\infty}\right), H^{0,0}\left(D_{\infty}\right)\right]_{1 / 2}\right)^{*} \\
& =\left[H^{0,0}\left(D_{\infty}\right),\left(H^{2,1}\left(D_{\infty}\right)\right)^{*}\right]_{1 / 2}
\end{aligned}
$$

(cf.[11], Vol.1, p.29) and for any

$$
g \in H^{1 / 2,1 / 4}\left(\partial D_{\infty}\right)=\left[H^{3 / 2,3 / 4}\left(\partial D_{\infty}\right), H^{-1 / 2,-1 / 4}\left(\partial D_{\infty}\right)\right]_{1 / 2}
$$

(cf. [11], Vol.2, p.70), there is a unique solution

$$
u \in H^{1,1 / 2}\left(D_{\infty}\right)=\left[H^{2,1}\left(D_{\infty}\right), H^{0,0}\left(D_{\infty}\right)\right]_{1 / 2}
$$

of the problem (5.1) depending continuously upon the data (cf. [11], Vol.2, p.78).

(4) Now from $u \in H^{1,1 / 2}\left(D_{\infty}\right)$, it follows that

$$
\Delta u \in H^{-1,0}\left(D_{\infty}\right) \text { and hence } u_{t}=f+\Delta u \in H^{-1,0}\left(D_{\infty}\right) .
$$

This proves Lemma 2 .

Lemma 3 The trace operator

$$
\gamma: W\left(\mathbb{R}_{\infty}^{n}\right) \rightarrow H^{1 / 2,1 / 4}\left(\partial \Omega_{\infty}\right)
$$

is continuous and onto.

Proof. Let $B$ denote a n-dimensional ball containing $\Omega$ properly. By Lemma 2 , with $f=0$, there is a vector field $u \in W\left((B \backslash \bar{\Omega})_{\infty}\right)$ such that

$$
\gamma_{\partial B} u=0, \quad \gamma_{\partial \Omega} u=g \in H^{1 / 2,1 / 4}\left(\partial \Omega_{\infty}\right) .
$$

We extend $u$ to a vector field $u \in W\left(\left(\mathbb{R}^{n} \backslash \bar{\Omega}\right)_{\infty}\right)$ with the help of the extension operator (of the Lemma 12.2 in [11], Vol.1, p.75). Applying this operator once more, we find that $u \in W\left(\mathbb{R}_{\infty}^{n}\right)$ with $\gamma u=g$. This implies the surjectivity of $\gamma$ and its continuity is obvious. 
From Lemma 3, we conclude that the injectivity of dual operator $\gamma^{*}$ from $H^{-1 / 2,-1 / 4}\left(\partial \Omega_{\infty}\right)$ onto a closed subspace of $W^{*}\left(\mathbb{R}_{\infty}^{n}\right)$ implies that

$$
\text { const. }\|\phi\|_{-1 / 2,-1 / 4} \leq\left\|\gamma^{*} \phi\right\|_{W^{*}\left(\mathbb{R}_{\infty}^{n}\right)} \leq \text { const. }\|\phi\|_{-1 / 2,-1 / 4}
$$

for all $\phi \in H^{-1 / 2,-1 / 4}\left(\partial \Omega_{\infty}\right)$. As a result, the coercivity of $A^{\prime}$ follows. More precisely, from (4.6), we see that

$$
\begin{aligned}
<\phi, A^{\prime} \phi> & =<\phi, \gamma L^{\prime-1} \gamma^{*} \phi> \\
& =<L^{\prime} L^{\prime-1} \gamma^{*} \phi, L^{\prime-1} \gamma^{*} \phi>, \quad \text { since } \gamma^{*} \phi \in H^{-1,-1 / 2}\left(\mathbb{R}_{\infty}^{n}\right) \\
& \geq \beta\left\|L^{\prime-1} \gamma^{*} \phi\right\|_{1,0}^{2} \quad \text { from }(4.11) \text { with } L^{\prime} \text { instead of } L^{*} \\
& \geq \text { const. }\left\|\gamma^{*} \phi\right\|_{W^{*}\left(\mathbb{R}_{\infty}^{n}\right)}^{2} \quad \text { from }(4.15) \\
& \geq \text { const. }\|\phi\|_{-1 / 2,-1 / 4}^{2} \quad \text { from (5.8) }
\end{aligned}
$$

Summarizing (4.19) and (5.9) now gives the first part of the following lemma.

Lemma 4 The operator

$$
A^{\prime}: H^{-1 / 2,-1 / 4}\left(\partial \Omega_{\infty}\right) \rightarrow H^{1 / 2,1 / 4}\left(\partial \Omega_{\infty}\right)
$$

is continuous and coercive. The same holds for the operator

$$
e^{-\lambda t} A: H^{-1 / 2,-1 / 4}\left(\partial \Omega_{T}\right) \rightarrow H^{1 / 2,1 / 4}\left(\partial \Omega_{T}\right)
$$

for any fixed $\lambda>0$.

It still remains to establish the coercivity of the operator $e^{-\lambda t} A$. We proceed as follows. For any $\phi \in H^{-1 / 2,-1 / 4}\left(\partial \Omega_{T}\right)$, we have

$$
\begin{aligned}
<\phi, e^{-2 \lambda t} A \phi> & =<e^{-\lambda t} \phi, R A^{\prime} E e^{-\lambda t} \phi>\quad \text { from }(4.21) \\
& =<E e^{-\lambda t} \phi, A^{\prime} E e^{-\lambda t} \phi> \\
& \geq \text { const. }\left\|E e^{-\lambda t} \phi\right\|_{H^{-1 / 2,-1 / 4}\left(\partial \Omega_{\infty}\right)}^{2} \quad \text { from }(5.9) \\
& =\text { const. }\left\|e^{-\lambda t} \phi\right\|_{H^{-1 / 2,-1 / 4}\left(\partial \Omega_{T}\right)}^{2} \\
& \geq \text { const. }\|\phi\|_{-1 / 2,-1 / 4}^{2}
\end{aligned}
$$

the last estimate following by duality. 


\section{Proof of Theorem 1}

The proof extends the standard variational technique to the unsteady integral equation. First, we will show that

$$
<\phi, A \phi>\geq \text { const. }\|A \phi\|_{H^{1 / 2,1 / 4}\left(\partial \Omega_{T}\right)}^{2}
$$

for all $\phi \in H^{-1 / 2,-1 / 4}\left(\partial \Omega_{T}\right)$. For this, the well-known jump relation of the simple-layer potential $V \phi$ may be written (cf. [9], 395 ff.)

$$
[\sigma(V \phi)]=\phi \quad \text { on } \quad \partial \Omega_{T}
$$

where the square brackets denote the difference between the interior and exterior trace: $[\sigma]:=\sigma_{i}-\sigma_{e}$ (see (2.6)). In the following, const. denotes a generic positive constant, and integrals are understood in a weak sense. By inserting (6.2) into (6.1), we obtain with $u=V \phi$,

$$
\begin{aligned}
<\phi, A \phi> & =<[\sigma(u)], A \phi> \\
& =\left\{\frac{1}{2} \int_{\mathbb{R}^{n}}|u(x, T)|^{2} d x+\int_{0}^{T} \int_{\mathbb{R}^{n}} \sum_{i, k}\left(\frac{\partial u_{i}}{\partial x_{k}}+\frac{\partial u_{k}}{\partial x_{i}}\right)^{2} d x d t\right\}
\end{aligned}
$$

By Korn's inequality,

$$
<\phi, A \phi>\geq \text { const. }\|u\|_{H^{1,0}\left(\mathbb{R}_{T}^{n}\right)}^{2}
$$

is implied by (6.3). Now since $u$ solves the differential equation (1.4) with $f=0$, an argument repeated the one employed in Section 4 shows that

$$
\|u\|_{H^{1,1 / 2}\left(\mathbb{R}_{T}^{n}\right)} \leq \text { const. }\|u\|_{H^{1,0}\left(\mathbb{R}_{T}^{n}\right)} .
$$

This together with the trace theorem ([11], Vol.2, 9f) gives (6.1).

Next, from the Proposition 1, an elementary argument shows that $A$ is an isomorphism. This means we have the estimates

$$
\text { const. }\|\phi\|_{-1 / 2,-1 / 4} \leq\|A \phi\|_{1 / 2,1 / 4} \leq \text { const. }\|\phi\|_{-1 / 2,-1 / 4}
$$

from which together with (6.1) follows the coercivity of A. This completes the proof of Theorem 1. 


\section{References}

[1] Arnold, D.N., and Noon, P.J., Coercivity of the single layer heat potential, J. Comput. Math, 7 (1989), 100-104.

[2] Costabel M., Boundary integral operators for heat equation, Integral Equations Operator Theory, 13 (1990), 489-552.

[3] Fichera, G., Simple layer potentials for elliptic equations of higher order, pp. 1-14 in Boundary integral methods, theorey and applications, Morino, L., and Piva, R. (ed.), Berlin 1991.

[4] Hebeker, F.K., The penalty method applied to the instationary Stokes equations, Applic. Anal. 14 (1982), 137-154.

[5] Hebeker, F.K., Characteristics and boundary elements for 3D nonstationary Navier Stokes equations, pp. 433-442 in Boundary Element IX, Vol.3, Brebbia, C.A., Wendland, W.L., and Kuhn, G. (ed.), Berlin 1987.

[6] Hebeker, F.K., and Hsiao, G.C., On Volterra boundary integral equations of the first kind for nonstationary Stokes equations, Advances in boundary element techniques, Springer Ser. Comput. Mech. Springer: Berlin, 1993, 173-186.

[7] Hsiao, G.C., and Saranen, J.,Boundary integral solution of the twodimensional heat equation, Math. Meth. Appl. Sci. 16 (1993), 87-114.

[8] Hsiao, G.C., and Wendland, W.L., Boundary element method: foundation and error analysis, Chapter 12 in Encyclopedia of Computational Mechanics, Edited by Erwin Stein, René de Borst and Thomas J.R. Hughes. Volume 1: Fundamentals, pp.339-373. John Wiley \& Sons, 2004

[9] Ladyzhenskaja, O.A., Solonnikov, V.A., Uralźewa, N.N., Linear and quasilinear equations of parabolic type, Providence 1968.

[10] Lions, J.L., Equations Differentielles Operationelles et Problémes aux Limites, Berlin etc. 1961.

[11] Lions, J.L., and Magenes, E., Non-homogeneous Boundary Value Problems and Applications, Vol.1-2, Berlin etc. 1972. 